\title{
La (traducción de la) historia como narrativa ${ }^{1}$
}

\section{The (translation of) history as narrative}

\section{Bárbara CERRATO RODRÍGUEZ ${ }^{2}$}

Universidad de Salamanca

cerrato@usal.es

Recibido: 5-4-2016. Revisado: 17-6-2016. Aceptado: 12-10-2016.

\begin{abstract}
The aim of this essay is to address the contemporary conception of translation and history as narratives of reality, influenced by the dominant Ideology and Power. As in the case of translation, in order to access the real history(ies) of the subalterns, first it is necessary to demystify the official History, drawn up by the official discourse of the ruling class. In this sense, particular attention should be paid to historiographic metafiction, since it is within the limits of historical discourse without relinquishing its autonomy as fiction, and has been utilized to reveal microhistories of the Other, subverting the official
\end{abstract}

1. El presente artículo se inscribe en el proyecto de investigación «Violencia simbólica y traducción: retos en la representación de identidades fragmentadas en la sociedad global» (FFI2015-66516-P), financiado por el Ministerio de Economía y Competitividad del Gobierno de España.

2. Becaria predoctoral del Programa para la Formación de Personal Investigador (FPI) del proyecto de investigación «Violencia simbólica y traducción: retos en la representación de identidades fragmentadas en la sociedad global» (FFI2015-66516-P; Convocatoria I+D+i 2016) financiado por el Ministerio de Economía y Competitividad del Gobierno de España y el Fondo Social Europeo. GIR «TRADIC» (Traducción, Ideología y Cultura) de la Universidad de Salamanca. 
discourse. Furthermore, it has been employed especially by women for ideological purposes, as it has helped them to reveal feminine duplicity, among other aspects.

Keywords: translation as narrative; history as narrative; historiographic metafiction.

Resumen: El propósito de este artículo es abordar la concepción contemporánea de la traducción y la historia como narrativas de la realidad, influidas por la Ideología y el Poder dominantes. Al igual que en el caso de la traducción, para acceder a la(s) historia(s) real(es) de los subalternos, primero es preciso desmitificar la Historia oficial, configurada mediante el discurso oficial de la clase dominante. En este sentido, la metaficción historiográfica merece una atención especial, puesto que se sitúa dentro de los límites del discurso histórico sin renunciar a su autonomía como ficción, y ha sido utilizada para sacar a la luz microhistorias del Otro, subvirtiendo el discurso oficial. Además, ha sido empleada con fines ideológicos especialmente por las mujeres, ya que les ha servido para revelar, entre otros aspectos, la duplicidad femenina.

Palabras clave: traducción como narrativa; historia como narrativa; metaficción historiográfica

History is, after all, a storying. Gayatri Spivak ([1993] 1996, 25)

\section{INTRODUCCIÓN}

Principalmente a partir de la década de los ochenta y los noventa del siglo XX, y gracias a la concepción de la traducción como fiel reflejo de los cambios sociales, políticos y culturales, se produjo una redefinición de la disciplina, a la que se incorporaron otros conceptos como manipulación (Hermans 1985), ideología y cultura (Bassnett y Lefevere 1990), violencia simbólica (Bourdieu [1998] 2015), microfísica del poder (Foucault 1985) y reescritura (Lefevere 1992). Así, los Estudios de Traducción comenzaron a abordar cuestiones postestructuralistas como la representación del Otro, el Poder occidental y su representación, la relación entre la producción del conocimiento y su transmisión, y su consiguiente admisión por la cultura meta.

El Poder resulta especialmente significativo, puesto que entiende que el discurso oficial, legitimado por la ideología dominante, se halla en la cúspide. Foucault relacionó por primera vez, en las décadas de los sesenta y los setenta, el lenguaje y el Poder (Foucault [1971] 1992, 19), una teoría que posteriormente recibió una gran acogida en los Estudios de Traducción (Lefevere y Bassnett [1990] 1995; Lefevere 1992). Además, Foucault explica la existencia del Poder organizado alrededor de su concepto de la microfísica, que defiende la existencia de múltiples focos de emisión de discursos cuya pretensión es convertirse en Verdades, lo que nos permite colegir que, dado que el lenguaje es una herramienta de Poder, también lo serán tanto la traducción como la historiografía en tanto que traducción y narrativa de la realidad, un concepto 
novedoso en nuestra disciplina que desarrollaremos en la segunda sección de este artículo. Por su parte, Bourdieu consideraba el Poder una vía para re-presentar la realidad y crear significados (1991; 1992): «and all construction of meaning implies acceptance of the power generating it» (Vidal 2017). Así, tiene lugar lo que Bourdieu denomina «el imperialismo del universal» $(1992,154)$, es decir, el hecho de que el Poder puede perpetuar o menoscabar el discurso que emana de las instituciones. Del mismo modo, Lefevere recurrió a la noción de Poder de Foucault para demostrar que escribir (la historia, por ejemplo) no es neutro, y tampoco lo es traducir. Y es que si algo ha quedado claro tras el giro cultural (Lefevere y Bassnett [1990] 1995) y el giro de poder (Tymoczko y Gentzler 2002) es que nuestra disciplina no es fiel ni neutral, sino que supone un posicionamiento ideológico deliberado (Tymoczko 2003) y se nutre de una toma de decisiones constante y deliberada (Tymoczko y Gentzler 2002, xxi) en la que el traductor participa del Poder y contribuye a crear el conocimiento:

In this conflict-ridden and globalized world, translation is central to the ability of all parties to legitimize their version of events, especially in view of the fact that political and other types of conflict today are placed out in international arena and can no longer be resolved by appealing to local constituencies alone (Baker 2006, 1).

Por lo tanto, puede que debamos cambiar nuestra cosmovisión y reconocer la existencia potencial y el valor de múltiples verdades (Baker 2006, 18-19), puesto que no debemos olvidar que:

Translation always implies an unstable balance between the power one culture can exert over another. Translation is not the production of one text equivalent to another text, but rather a complex process of rewriting that runs parallel both to the overall view of language and of the 'Other' people have throughout history; and to the influences and the balance of power that exist between one culture and another (Álvarez y Vidal 1996, 4).

\section{EL CONCEPTO DE HISTORIA COMO NARRATIVA EN LA ACTUALIDAD}

La escritura de la Historia se consideró un ámbito de la Retórica hasta finales del Neoclasicismo, cuando la Historia empezó a erigirse como una Episteme en sí misma (Gossman 1978, 3-5). Este cambio de concepción implicaba que la disciplina era objetiva y que se iba desarrollando gracias a la investigación científica. Leopold von Ranke, impulsado por el positivismo, fue el precursor de la historiografía moderna a comienzos del siglo XIX, al exponer que el fin último que motivaba sus investigaciones 
eran la objetividad y la neutralidad científicas: de hecho, afirmó que quería «bloss zeigen, wie es eigentlich gewesen»³ ([1824] 2012). Hoy en día, además, algunos diccionarios contemporáneos siguen definiendo la Historia como ciencia, como el Diccionario Salamanca ${ }^{4}$.

Pese a lo anterior, el carácter científico de la Historia empezó a cuestionarse en el siglo XX, a partir de la sociología de Emile Durkheim: "[p]or primera vez se negaba el carácter científico, y por lo tanto único y objetivo, de la historia y la idea de que se pudieran formular leyes a partir de los acontecimientos del pasado» (Vidal, en prensa): «[t]o the question 'What is history?', we must always add: 'Who decides? On what grounds, and to what end?'» (Morley 1999, 23). No obstante, el realismo histórico ya se había puesto en tela de juicio a principios del siglo XIX, cuando algunos expertos empezaron a ser conscientes de que la objetividad no era tal, dado que quienes construían el conocimiento estaban implicados necesariamente en el proceso y porque, además, las narrativas históricas se construían gracias al lenguaje (Vidal, en prensa) ${ }^{5}$. En este sentido, destacan especialmente las Tesis sobre la filosofía de la historia ([1955] 2005), de Benjamin ${ }^{6}$.

Este texto merece especial atención, en tanto que, en él, Benjamin intentó revelar las limitaciones de la visión rankeana de la historia como ciencia objetiva. De hecho, Benjamin afirma en su Tesis IV que historiar el pasado no significa hacerlo «tal y como fue realmente», según defendía Ranke, sino más bien lo contrario: «adueñarse de un recuerdo tal y como relumbra en el instante de un peligro» (Benjamin [1955] 2005, 7). Por eso, sólo se deben recuperar ciertos sucesos relativos, "constelaciones cargadas de tensiones» (Tesis XVII, ibid., 15), a un suceso determinado o, de lo contrario, ello supondrá «prestarse a ser instrumento de la clase dominante» (ibid., 7). Es decir, Benjamin aboga por descubrir múltiples pasados, y no sólo el del vencedor:

La naturaleza de esa tristeza se hace patente al plantear la cuestión de con quién entra en empatía el historiador historicista. La respuesta es innegable que reza así: con el vencedor. [...] Como suele ser costumbre, en el cortejo triunfal llevan consigo el botín. Se lo designa como bienes de cultura. [...] Jamás se da un documento de cultura sin que lo sea a la vez de la barbarie (Tesis VIl; ibid., 8-9).

3. «[Quería] mostrarlo tal y como fue realmente» (mi traducción).

4. Véase la versión electrónica de este diccionario: http://fenix.cnice.mec.es/diccionario/.

5. «[H]istory constructs its objects, and [...] its objects are objects of language, rather than entities of which words are in some way copies» (Gossman 1978, 29).

6. Es preciso destacar también los intentos por empezar a narrar la historia desde el principio de las ciencias sociales, el marxismo, la Escuela de los Annales y la publicación del Journal of the History of Ideas en 1940 (Vidal, en prensa).

Bárbara CERRATO RODRIGUEZ La (traducción de la) historia como narrativa 
Para sacar a la luz estos pasados «vencidos, que, a pesar de haber sido derrotados, continúan vivos y actuantes, determinando una parte muy importante de la historia, subterránea y reprimida pero presente dentro del devenir histórico» (Aguirre Rojas 2006, 40), Benjamin sostiene que hay que pasar «el cepillo a contrapelo» sobre la historia (Tesis XVII):

Porque sólo al avanzar a contracorriente de esa historia rehecha por las clases dominantes, será posible restituir esos pasados derrotados y esos proyectos y líneas en conflicto, que después del combate han resultado sólo provisionalmente avasallados y borrados. Avance a contrapelo de la historia oficial y dominante que nos hará posible desmitificar los "orígenes gloriosos", y también las genealogías siempre vencedoras y conquistadoras que llenan esas historias oficiales, a la vez que nos permite deslegitimar la continuidad siempre positiva, ascendente, gloriosa y supuestamente indetenible del avance y el triunfo de los actuales dominadores (Echeverría 2005).

De este modo, para acceder a la historia real, primero es preciso deslegitimar y desmitificar la Historia oficial, configurada a través de la negación y el sometimiento de las clases dominadas. Y es que la negación de la historia del Otro supone, asimismo, la negación de su identidad.

Años más tarde, durante las décadas de los sesenta y los setenta del siglo XX, otros historiadores, como Hayden White, Dominick LaCapra, Michel de Certeau, Paul Veyne, Peter Burke, Louis O. Mink, Lionel Gossman, David Carr, Maurice Mandelbaum, W. H. Dray, Nöel Carroll, Frank Ankersmit o Jacques Rancière entendían la historia como un proceso "of authorial insight, invention and experimentation» (Munslow 2013, 3), desechando con ello la definición clásica, y por consiguiente la objetividad y la neutralidad asociadas a ella, al igual que hicieran algunos expertos durante el giro lingüístico de la Traducción. Este paralelismo es precisamente la hipótesis de partida de nuestro estudio, algo apuntado por algunos autores ya consagrados, como Hayden White, que advierte sobre la idea de que el historiador es un traductor de la realidad, una teoría que apenas ha sido desarrollada en nuestra disciplina ${ }^{7}$.

Translation is in history, always. It is, in many cases, a vital factor within history, and the more we learn about its history, the more obvious this fact becomes. It is no coincidence, therefore, that many histories of translation have been published over the last ten years, just as it is no exaggeration to say that if we want to study cultural history, the history of philosophy, literature, and religion, we shall have to study translations to a much greater extent than we have done in the past (Bassnett y Lefevere 1998, 6).

\section{Véase también Vidal en Vorderobermeier (2013).}

Bárbara CERRATO RODRIGUEZ La (traducción de la) historia como narrativa 
En vista de todo ello, parece oportuno detenernos brevemente en Lawrence Stone y Michel de Certeau. El primero desarrolló una forma nueva de ver la historia: "se fijaba más en la cultura de un grupo o en la voluntad de los individuos y menos en la posibilidad de una racionalidad científica de los hechos históricos» (Stone 1979), si bien parece que nunca llegó a renunciar a la racionalidad y la reconstrucción realista del pasado. El segundo, de Certeau ${ }^{8}$, asoció la historiografía con la representación del Otro y la cuestión del Poder occidental:

[P]or eso comienza su magnífico libro titulado The Writing of History con el conocido cuadro de Jan Van der Straet de principios del siglo XVII en el que se representa alegóricamente a Américo Vespucio despertando a América. Se trata de un cuadro con connotaciones colonialistas que también han analizado otros intelectuales, porque a partir de él se entiende la escritura de la historia como un acto de dominación, como una escritura de conquista; el Nuevo Mundo como un espacio en blanco, como una página «salvaje» en la que desea escribir el occidental (Vidal, en prensa).

Es decir, en opinión de de Certeau, la historia es una suerte de (re)traducción del pasado que tiene significado gracias al lenguaje:

Historiography (that is, «history" and "writing") bears within its own name the paradox -almost an oxymoron- of a relation established between two antinomic terms, between the real and discourse. Its task is one of connecting them and, at the point where this link cannot be imagined, of working as if the two were being joined. [...] From this standpoint, reexamination of the historiographical operation opens on the one hand onto a political problem (procedures proper to the «making of history» refer to a style of «making history») and, on the other, onto the question of the subject (De Certeau [1975] 1988, xxvii).

Como ya hemos apuntado, Foucault parte del enorme poder del discurso que emana de las instituciones y que se va filtrando subrepticiamente y modelando microfísicamente el mundo y las distintas identidades. Así, propone elaborar una contrahistoria y una contramemoria, en contraposición al discurso oficial de la Historia, y recomienda sospechar de la existencia de luchas, victorias, heridas, dominaciones y servidumbres en el corazón de las palabras (Foucault [1970] 1987, 15). De este modo, es necesario llevar a cabo una genealogía de la Historia basada en su discontinuidad: "[l]os discursos deben ser tratados como prácticas discontinuas que se cruzan, a veces

8. De Certeau acuña el término heterología: hetero-, en alusión al Otro, y -logía, refiriéndose al discurso ([1986] 2010): «es esa ambigüedad que existe entre el discurso sobre el otro y el discurso del otro" (Vidal, en prensa). 
se yuxtaponen, pero que también se ignoran o excluyen», por lo que «[e]s necesario concebir el discurso como una violencia que se ejerce sobre las cosas» (ibid., 53).

En esta misma línea, Vidal sostiene que la Historia oficial ha asignado identidades e impuesto una posición, «la de la institución, que se entiende como punto de vista legítimo, es decir, como punto de vista que todo el mundo debe aceptar por lo menos dentro de los límites de una sociedad determinada» (en prensa). En palabras de Bourdieu, las instituciones, que se erigen como portadoras de la Verdad universal y el Poder absoluto, ejercen una violencia simbólica legítima ([1985] 2008). En vista de todo lo anterior, y tal y como afirma Munslow en el prefacio al libro de Jenkins ([1991] 2003, xi), cabría colegir entonces que las historias son:

[O]nly histories. This means we would do well to recognize and remember that the histories we assign to things and people are composed, created, constituted, constructed and always situated literatures. And, what is more, they carry within them their author's philosophy or 'take' on the world present, past and future. [...] 'history' is not the same as 'the past'. By the same token that as a form of knowledge history is -plainly and palpably- a narrative representation (id.).

Según esta dirección investigadora de la historiografía, y pese a los esfuerzos invertidos en lo que Hayden White $(1987,20)$ ha denominado la «doxa del establishment historiográfico" ${ }^{9}$, es preciso reconocer que la historia es el conocimiento del pasado y la manera de acceder a él. De este modo, la clave residiría entonces en el concepto de conocimiento mismo y en tener en cuenta la base epistemológica, filosófica y ontológica que guía al investigador (Vidal, en prensa).

$[\mathrm{H}]$ istory is always for someone [...] history always has a purpose [...] history is always about power [...] history is never innocent but always ideological (and not in the sense of political bias, but moral judgements about right and wrong and how the individual historian thinks the world works (Munslow en Jenkins [1991] 2003, xiii).

No obstante, resulta oportuno señalar que los historiadores mencionados anteriormente no buscan negar el conocimiento histórico, sino poner de relieve que la historia, al igual que la traducción, es un sistema de significación en el que «the meaning and shape are not in the events, but in the systems which make those past 'events' into present historical 'facts'. This is not a 'dishonest refuge from truth' but an acknowledgement of the meaning-making function of human constructs» (Hutcheon

9. Podría definirse como «una perspectiva que intentaba borrar las huellas del sujeto, el rastro de sus circunstancias particulares, y que entendía por tanto que el discurso carecía de sesgo subjetivo alguno y que el relato era equivalente a la estructura misma de los hechos» (Vidal, en prensa).

Bárbara CERRATO RODRIGUEZ La (traducción de la) historia como narrativa 
1988, 89). Asimismo, pretenden reivindicar que la historia es una lectura individual del pasado: el historiador, en tanto que ser humano, selecciona los hechos, los interpreta y los narra (Morley 1999, 60 ss.). De hecho, algunos de estos historiadores le otorgan un peso significativo a la traducción, pues les sirve como vía para (re)traducir el pasado:

[W] can come to understand the ancient world (and any other period of history, and any other culture) only through translation, finding equivalents in our language for their words, concepts and ideas. Inevitably, there is a risk of missing something important, or introducing something new by mistake, but this risk is inherent in every attempt at understanding the past (ibid., 81).

A continuación, exploraremos brevemente el continuum entre la historia y la literatura, la metaficción historiográfica, puesto que varios escritores han recurrido a ella para componer novelas historiográficas (re)traduciendo la historia desde los ojos del Otro.

\section{EL CONTINUUM ENTRE LA(S) HISTORIA(S) Y LA LITERATURA: LA METAFICCIÓN HISTORIOGRÁFICA}

La existencia de una única Historia trae consigo la aparición de estereotipos, estereotipos que Adichie considera incompletos, puesto que «[t]hey make one story become the only story» (2009). Según esta misma autora, la consecuencia principal de que exista una única Historia, que jamás es accidental, es que las personas se ven despojadas de su dignidad:

It robs people of dignity. It makes our recognition of our equal humanity difficult. It emphasizes how we are different rather than how we are similar. [...] It is impossible to talk about the single story without talking about power. [...] Power is the ability not just to tell the story of another person, but to make it the definitive story of that person (id.).

El Poder -colonial, sexista, racista...- ha configurado a su antojo las traducciones del mundo mediante narrativas coherentes y «naturales», recordando el término que emplea Barthes en Mitologías ([1957] 1999). Por consiguiente, parece que el traductorhistoriador debería plantearse numerosas preguntas antes de abordar un texto, sobre todo acerca de la intención y la ideología que han motivado su redacción, el hecho de que se hayan escogido unas palabras y no otras, y un largo etcétera. Además, puede que también deba preguntarse a quién beneficia o perjudica el texto o si intenta deconstruir o perpetuar las oposiciones binarias entre la mayoría y la minoría. En este sentido, Vidal recomienda preguntarse también «en relación con qué es marginal 
lo marginal», pese a que admite que se trata de una cuestión «difícil de responder porque el Poder se ejerce normalmente desde la microfísica, desde un centro que muchas veces no quiere dejarse descubrir con facilidad pero que quiere universalizar, homogeneizar, sus ideas como algo 'natural'» (en prensa).

When we say marginal, we must always ask, marginal to what? But this question is difficult to answer. The place from which power is exercised is often a hidden place. When we try to pint it down, the center always seems to be somewhere else. Yet we know that this phantom center, elusive as it is, exerts a real, undeniable power over the whole social framework of our culture, and over the ways that we think about it. [...] The need to enforce values which are at the same time alleged to be «natural» demonstrates the insecurity of a center which could at one time take its own power much more for granted (Ferguson en Ferguson et al. 1990, 9-10).

Como decíamos, se trata de una tarea harto compleja, pues el discurso oficial suele tildar las narrativas subalternas de «dislocaciones» de las narrativas dominantes ${ }^{10}$ (Said 1986, 140). En vista de todo lo anterior, y como dejamos entrever antes, parece que actualmente ya no es posible hablar de la Historia, sino de historias en plural (Gardiner 1988). Justamente en esta línea de investigación, la École des Annales propuso comenzar a explorar la nouvelle histoire, (re)orientada hacia la microhistoria, "que observa las realidades pequeñas frente a la mirada amplia y macroestructural de los franceses, y rompe con las formas autoritarias e impositivas del discurso historiográfico tradicional que presenta la realidad como objetiva» (Vidal, en prensa). En definitiva, tanto la microhistoria como la historiografía crítica buscan reescribir la historia «desde abajo» (Thompson 1966; Kantz 1988; Burke 1991) y la Alltagsgeschichte, dando voz a los subalternos y la gente ordinaria: "[g]one is the focus on the 'great men' of politics and world affairs and in comes the focus on 'ordinary' people as a group» (Munday 2014, 67).

Por otro lado, en lo que respecta a los Estudios de Traducción, algunos teóricos entienden la disciplina a modo de narración, una línea de investigación de todo punto novedosa. A modo de ejemplo, Baker (2006) toma como punto de partida a Barthes y Foucault para concebir la traducción como narración en tanto que construcción y representación de la realidad. Del mismo modo, esta nueva línea de investigación incorpora temas como la otredad, la manipulación, el poder y la ideología a la hora de hacer historias de la traducción (Bastin y Bandia 2006, 2). Ello se debe a que la actividad traductora del Otro también ha sido objeto de negación, esgrimiendo como excusa que sólo existía la tradición oral (Bandia 2009, 209). Según Bandia, para llevar a cabo estas historias de la traducción, son precisos «research paradigms such as power relations

10. Según Said $(1986,140)$, «[w]henever we try to narrate ourselves, we appear as dislocations in their discourse».

Bárbara CERRATO RODRIGUEZ La (traducción de la) historia como narrativa 
and ideology, sociology and transculturality, gender and postcoloniality» (en Bastin y Bandia 2006, 47). En opinión de este mismo autor, la superación de las perspectivas androcéntricas y eurocéntricas sería un buen comienzo para interrelacionar la historia y la traducción, y comenzar una nueva línea de investigación en historia de la traducción que sea mucho más inclusiva y tenga en cuenta las relaciones entre Oriente y Occidente (2006, 51-52). En definitiva, se persigue una historia de la traducción:

that accounts for marginalised subjects and contexts would draw attention to minoritised contexts and tradition that are often overlooked in the global marketplace of knowledge. Translation history must account for the power inequalities inherent in global relations by shifting attention away from the dominant metropolitan cultures and canonical subjects to include those marginalized cultures that have been consigned to the periphery by forces of imperialism and colonization (Bandia 2014, 117).

En esta misma línea, resulta especialmente relevante el monográfico de la prestigiosa revista The Translator (20: 1, 2014) dedicado a estas nuevas formas de hacer historia de la traducción a partir de las nuevas directrices historiográficas a las que nos referíamos antes. Asimismo, el artículo de Munday publicado en dicho monográfico es muy significativo para el propósito del presente artículo, ya que aplica los puntos de la microhistoria a las nuevas formas de hacer historia de la traducción. Según este autor, es necesario tener en cuenta las circunstancias de los traductores porque éstas determinan sus historias, y es que todo depende del tipo de historia que queramos contar (Munday 2014, 66). Se trata, en definitiva, de fusionar microhistorias y macrohistorias relacionadas para lograr una historia de la traducción más inclusiva (Bandia 2014, 117). Sin embargo, no debemos olvidar que, para que esta(s) microhistoria(s) puedan salir a la luz, primero pasan por el tamiz de quien las selecciona, bien un traductor bien un historiador: «these new narratives of microhistory also conceal other biases in the selection and presentation of material, in the gaps of our knowledge and in forms of overtly mediated testimonies» (ibid., 77).

Es decir, en este punto, la historiografía y la traductología confluyen: «translation, as an intellectual activity, can be put to the service of history by allowing us to search deep into the past to rectify facts and hopefully avoid scholarly distortions» (Bandia 2009, 226). La primera entiende al historiador como traductor y la segunda, al traductor como historiador. Del mismo modo, ambas se basan actualmente en la concepción anti-rankeana de la historia y adoptan el enfoque foucaultiano que concibe la historia como un discurso de Poder influido por las ideologías de las instituciones. Asimismo, ambas sostienen que la realidad es producto del lenguaje, un instrumento de Poder: "[n]o event is recounted in an identical manner by two different actors, which is clearly a substantial problem for a branch of the discipline that seeks to construct a scientific or empirical base» (Munday 2014, 66). Por tanto, el traductor-historiador debe ser 
consciente del poder de la palabra y valorar cuestiones como qué es lo que se traduce, por qué se traduce un determinado texto, quién decide lo que se debe traducir, etc. Y desde el punto de vista de la historia de la traducción: «the question is What is the role of the historian in recreating the past? " (Bastin en Bastin y Bandia 2006, 48).

De este modo, ambas disciplinas viran hacia los subalternos, es decir, hacia aquellos cuya voz ha sido silenciada por el Poder. En definitiva, parece que esta nueva historia se interesa ahora por lo local y, para ello, da voz a quien no la tenía pero que jamás renunció a ella, al subalterno, al Otro. Asimismo, para reelaborar esta nueva historia y empezar "desde abajo" (Thompson 1966; Kantz 1988; Burke 1991), se parte de las opiniones y las experiencias de la gente corriente acerca del cambio social y, para ello, se emplean otro tipo de fuentes, como la historia oral, las encuestas judiciales, los interrogatorios, etc. (Vidal, en prensa; Munday 2014, 66): "[w]hile requiring careful evaluation, such sources offer valuable testimony and more direct access to the working practices of the translator and can give crucial insights into both historical circumstance and translation» (Munday 2014, 66). Y es que cabría afirmar que en este sentido la historia es análoga a la traducción, pues ambas tienen el Poder de dar voz a los olvidados, los subalternos, como sostienen Spivak (1994) y los Subaltern Studies, y también a los marginados entre los cuales Munday incluye a los traductores $(2014,66)$-. Del mismo modo, ambas disciplinas se prestan a múltiples lecturas e interpretaciones, todas ellas enriquecedoras, que no hacen sino completar el original:

El pasado, como las traducciones, nunca se agota con una sola lectura o con la interpretación que alguien haya hecho de unos determinados hechos. La historia estará tanto más completa cuantas más voces se hayan incorporado a su construcción, igual que las traducciones se deben revisar porque envejecen, porque las sociedades y los contextos evolucionan y porque tal vez el traductor, en una primera interpretación, no haya visto todas las voces que hay detrás de cada texto (Vidal, en prensa).

Este cambio de perspectiva que han adoptado la historiografía y la traducción también se ha reflejado en la literatura, de forma que «muchos escritores se han sentido profundamente atraídos por esta perspectiva y han escrito novelas historiográficas que cuentan la historia desde la perspectiva del subalterno» (id.). A modo de ilustración, cabría citar A History of the World in 10 1/2 Chapters, de Barnes (1989), donde el autor sostiene que la historia no es lo que sucedió, sino lo que cuentan los historiadores que sucedió:

History isn't what happened. History is just what historians tell us. There was a pattern, a plan, a movement [...] it is a tapestry, a flow of events, a complex narrative, connected, explicable. One good story leads to another [...] all the time it's connections, progress, meaning, this led to this, this happened because of this. And we, readers of history, the sufferers from history, we scan the pattern for hopeful conclusions, for the

Bárbara CERRATO RODRIGUEZ La (traducción de la) historia como narrativa 
way ahead [...] We think we know who we are, though we don't quite know why we're here, or how long we shall be forced to stay. And while we fret and writhe in bandaged uncertainty [...] we fabulate. We make up a story to cover the facts we don't know or can't accept; we keep a few true facts and spin a new story around them. Our panic and our pain are only eased by soothing fabulation; we call it history (ibid., 242).

Como decíamos, la historia es tanto el conocimiento como los significados que construyen los historiadores a través del lenguaje. A modo de ejemplo, cabría referirse a la entrada elaborada por Luis Suárez sobre Francisco Franco en el Diccionario biográfico español editado por la Real Academia de la Historia (RAH) en 2001. Este medievalista español, antiguo Rector de Universidad, procurador en Cortes y Director General de Universidades durante la dictadura franquista y presidente actual de la Hermandad del Valle de los Caídos, es autor de la obra Franco y su tiempo, publicada en 1984, y para la cual el historiador contó con el acceso, prácticamente en régimen de exclusividad, a los fondos de la Fundación Francisco Franco. Así, Suárez califica al dictador de «autoritario, no totalitario" y le atribuye el calificativo de "Generalísimo o Jefe de Estado» $(2011,607)$, con las consiguientes connotaciones fascistas que evoca ese título en España, como nos recuerda Vidal $(2013,208)$. Del mismo modo, el historiador hace hincapié en el "frío valor que sobre el campo desplegaba» y su «incomparable valor, dotes de mando y energía desplegada en el combate», y lo califica de «inteligente y moderado» (Suárez 2011, 607). Además, según Suárez, Franco fue un «jefe riguroso y eficaz» que participó en una «guerra larga de casi tres años que le permitió derrotar a un enemigo que en principio contaba con más fuerza», en alusión a la cruenta guerra civil española (id.). Asimismo, señala que a Franco siempre le guió el «firme propósito de respetar y acatar la soberanía nacional», lo cual resulta especialmente llamativo en tanto que a Juan Negrín, Presidente de la República, se le tacha de «golpista» en su correspondiente entrada. Es evidente que ésta es tan sólo una de las traducciones posibles de la historia. De haber sido otro historiador quien hubiese escrito la entrada, el resultado podría haber sido radicalmente distinto. Vidal sugiere, por ejemplo, al historiador Paul Preston, «quien lleva años investigando sobre la Segunda República, la guerra civil y la dictadura desde una perspectiva muy diferente, tal y como aparece en libros suyos como Franco, 'Caudillo de España' (2006), El gran manipulador. La mentira cotidiana de Franco (2008) o El holocausto español (2011)» (2013, 209). A pesar de la polémica que suscitaron ésta y alguna otra entrada, la RAH se negó en un primer momento a hacer ninguna modificación ${ }^{11}$. No obstante, tras el informe que elaboró la comisión -formada por Carmen Iglesias, actual directora de la RAH,

11. Véase Lucas, Antonio y Argote, Álvaro (30/05/2011) «La Real Academia de la Historia 'no corregirá' la polémica biografía de Franco. El Mundo. Disponible en: http://www.elmundo.es/ elmundo/2011/05/30/cultura/1306744704.html.

Bárbara CERRATO RODRIGUEZ La (traducción de la) historia como narrativa 
Carmen Sanz Ayán, Vicente Pérez Moreda y Faustino Menéndez Pidal- encargada por la Real Academia, y forzada al menos parcialmente por el ministro de Educación en aquel momento, Ángel Gabilondo, y por la suspensión de la subvención pública que exigieron algunos grupos parlamentarios, la RAH cambió de parecer y anunció el año pasado que modificaría la entrada acerca del dictador ${ }^{12}$.

A continuación, nos referiremos brevemente a la metaficción historiográfica, cuyos partidarios suelen rechazar la oposición entre la ficción y la historia, puesto que apuntan que los historiadores tienen el Poder de silenciar historias del pasado -al igual que sostenían White, LaCapra, de Certeau, etc., según hemos visto antes-y dejan entrever que eso es justamente lo que han hecho (Hutcheon 1988; 1989). Según Hutcheon, la metaficción historiográfica es difícil de definir, pero lo hace de la siguiente manera: "[w]hat we tend to call postmodernism in literature today is usually characterized by intense self-reflexivity and overtly parodic intertextuality. In fiction this means that it is usually metafiction that is equated with the postmodern» (Hutcheon 1989, 3). Hutcheon completa esta definición con «an equally self-conscious dimension of history» (id.). Prosigue, además, ligando el postmodernismo con la metaficción historiográfica:

The term postmodernism, when used in fiction, should, by analogy, best be reserved to describe fiction that is at once metafictional and historical in its echoes of the texts and contexts of the past. In order to distinguish this paradoxical beast from traditional historical fiction, I would like to label it «historiographic metafiction» (id.).

En vista de lo anterior, parece que deben considerarse novelas de metaficción historiográfica todas aquellas cuya autorreflexión metaficticia (e intertextualidad) convierta sus pretensiones de veracidad histórica en algo problemático (id.). A modo de ilustración, Hutcheon cita las siguientes: Cien años de soledad (1967), de García Márquez, Ragtime (1975), de Doctorow, La mujer del teniente francés (1969), de Fowles, y El nombre de la rosa (1980), de Eco. A estas novelas, creo que sería preciso añadir The Wild Girl, de Roberts ${ }^{13}$. También a modo de ejemplo, esta misma autora se refiere al intento de la ficción americana de abrirse a la historia, al «mundo", en palabras de Said (1983), que no ha dado sus frutos, puesto que parece que es imposible hacerlo de un modo inocente (Hutcheon 1989, 4). Cabría colegir entonces que la metaficción historiográfica se sitúa dentro de los límites del discurso histórico sin renunciar a su autonomía como ficción, una suerte de parodia irónica para Hutcheon:

12. Véase [No hay datos sobre el periodista] (07/04/2015) «Carmen Iglesias a la cadena SER: 'Franco fue sin duda un dictador'». El País. Disponible en: http://cultura.elpais.com/cultura/2015/04/07/actualidad/1428402974 723203.html.

13. Véase Cerrato Rodríguez, B. (2016) «La traducción feminista intralingüística en The Wild Girl», Entreculturas, 9: 95-105. 
And it is kind of seriously ironic parody that effects both aims: the intertexts of history and fiction take on parallel (though not equal) status in the parodic reworking of the textual past of both the "world" and literature. The textual incorporation of theses intertextual past(s) as a constitutive structural element of postmodernist fiction functions as a formal marking of historicity - both literary and «worldly» (id.).

Lo cierto es que, tradicionalmente, bien se han "robado» las historias bien se han considerado propiedad común de una cultura o comunidad y «[t]hese notable happenings, imagined or real, lay outside language the way history itself is supposed to, in a condition of pure occurrence» (Gass 1985, 147). Por su parte, Hutcheon defiende que actualmente se ha retomado la idea de la «propiedad» discursiva común porque tanto los textos literarios como los históricos están engastados en la ficción. Sin embargo, reconoce que se trata de una afirmación problemática en tanto que la historia y la literatura se consideran constructos e ilusiones humanos, ya que la parodia intertextual ofrece la sensación de la presencia del pasado, pero es un pasado que sólo se puede conocer por sus textos, sus vestigios, bien sean literarios o históricos (Hutcheon 1989, 4):

Clearly, then, what I want to call postmodernism is a paradoxical cultural phenomenon, and it is also one that operated across many traditional disciplines. In contemporary theoretical discourse, for instance, we find puzzling contradictions: those masterful denials of mastery totalizing negations of totalization, continuous attestings of discontinuity. In the postmodern novel the conventions of both fiction and historiography are simultaneously used and abused, installed and subverted, asserted and denied (ibid., 4-5).

El texto se sitúa en el «mundo» del discurso, de los textos y los intertextos, pero, en definitiva, se trata de un «mundo» que, pese a tener vínculos directos con la realidad empírica, no deja de ser una lectura, una representación, que no equivale en absoluto al realismo en sí. Por consiguiente, las aspiraciones de la metaficción historiográfica podrían resumirse en dos: la primera, cuestionar el concepto de representación y la segunda, refutar la división categórica entre el arte y el mundo (real).

The frontiers of a book are never clear-cut: beyond the title, the first lines, and the last full stop, beyond its internal configuration and its autonomous form, it is caught up in a system of references to other books, other texts, other sentences: it is a node within a network (Foucault [1969] 1972, 23).

Entonces, parece que una obra no puede considerarse original porque, de lo contrario, carecería de sentido para el lector. De este modo, la intertextualidad (Barthes 
1977, 160; Riffaterre 1984, 142-143) sustituye la relación autor-texto por lectortexto, es decir, una relación que sitúa el locus del significado textual en la historia del discurso en sí: «[i]t is only as part of prior discourses that any text derives meaning and significance» (Hutcheon 1989, 7). Derrida, por su parte, ha llevado esta intertextualidad al extremo, logrando así romper con la totalidad de los contextos dados, generando con ello una infinidad de nuevos contextos ([1972] 1977, 185). Asimismo, la intertextualidad defiende la provisionalidad de los textos: asume que las prácticas discursivas anteriores se filtran inevitablemente. Es decir, la intertextualidad proporciona y mina el contexto, una paradoja que Leitch explica así:

[lt] posits both an uncentered historical enclosure and an abysmal decentered foundation for language and textuality; in so doing, it exposes all contextualizations as limited and limiting, arbitrary and confining, self-serving and authoritarian, theological and political. However, paradoxically formulated, intertextuality offers a liberating determinism (Leitch 1983, 162).

Dadas las circunstancias de esta intertextualidad, definida por Barthes como la imposibilidad de vivir fuera del texto infinito $(1975,36)$, la metaficción historiográfica aparentemente implica que el lector debe ser capaz de reconocer no sólo la textualidad inevitable de nuestro conocimiento del pasado, sino también el valor y los límites de esa forma de conocimiento discursivo (Hutcheon 1989, 8), pues está situado «entre la presencia y la ausencia», en palabras de Barilli (1974).

Por eso, según estos autores, la metaficción historiográfica se corresponde, al menos hasta cierto punto, con la idea de la historiografía como narrativización (en lugar de representación) del pasado (Hutcheon 1989, 8): "there is no one writable 'truth' about history and experience, only a series of versions: it always comes to us 'stencillized'» (Tanner 1971, 172). De este modo, no cabe borrar, sino más bien subrayar, la línea ontológica que divide en pasado histórico y la literatura (Thiher 1984, 190).

Sin embargo, este vínculo entre la intertextualidad y la narrativización no se debe considerar una limitación del enfoque ni del valor de la ficción, sino una expansión de los mismos. Asimismo, y sobre todo en el postmodernismo americano, la parodia intertextual se centra en lo "diferente» en términos de nacionalidad, etnia, género, raza u orientación sexual, y hace uso y abuso de los clásicos canónicos introduciendo cambios significativos en la cultura europea dominante -blanca, masculina y de clase media- (Hutcheon 1989, 12)

De hecho, desde los años sesenta, parece que la ficción americana ha estado obsesionada con su propio pasado literario, social e histórico (Bradbury 1983, 186). Por su parte, D'haen $(1986,216)$ sostiene que ello se debe a su necesidad por encontrar la voz americana en la tradición eurocéntrica dominante: «[t]he canonical

Bárbara CERRATO RODRIGUEZ La (traducción de la) historia como narrativa 
texts of the American tradition are both undermined and yet drawn upon, for parody is the paradoxical postmodern way of coming to terms with the past» (Hutcheon 1989, 14).

Finalmente, es preciso señalar que la metaficción historiográfica ha sido empleada con fines ideológicos, como cualquier otro tipo de texto, especialmente por las mujeres: «[p]arody for them is more than just a key strategy through which 'feminine duplicity' is revealed [...], though it is one of the major ways in which women both use and abuse, set up and then challenge male traditions in art» (ibid., 19).

\section{CONCLUSIONES}

Como decíamos al principio, según esta nueva línea de investigación, la traducción, y por ende la traducción de la historia, están influidas por tensiones y conflictos motivados por la Ideología y el Poder dominantes. Y es que está claro que la vida es una sucesión de traducciones, pues cada uno de los actos que realizamos está impregnado de nuestro bagaje y nuestra forma de ver el mundo, por lo que cabe argumentar que pueden existir tantas narrativas como personas habitan el planeta. Según este punto de vista, dedicarse a traducir (la realidad) es una tarea harto compleja, pues el lenguaje jamás es inocente, sino, antes bien, un elemento performativo que vive en tanto que hace. Por consiguiente, tanto el traductor como el historiador, en calidad de traductor de la Historia, deben posicionarse y decidir qué narrativa quieren favorecer: bien perpetuar el discurso oficial de los vencedores, de los opresores; bien dar voz a quienes no la han tenido hasta el momento, a los vencidos, a los oprimidos.

\section{REFERENCIAS BIBLIOGRÁFICAS}

ADICHIE, Chimamanda Ngozi. 2009. "The Danger of a Single Story», TED. Ideas Worth Spreading (web). Fecha de acceso 8 de marzo de 2016. https://www.ted.com/talks/chimamanda adichie the danger of a single story/transcript.

AGUIRRE ROJAS, Carlos Antonio. 2006. Retratos para la historia. Ensayos de contrahistoria intelectual. México: Contrahistorias.

ÁlvareZ, Román y Vidal, M. ${ }^{a}$ Carmen ÁFRICA. 1996. Translation, Power, Subversion. Clevedon: Multilingual Matters.

BAKER, Mona. 2006. Translation and Conflict: A Narrative Account. Londres/Nueva York: Routledge.

BANDIA, Paul F. 2009. "Cheikh Anta Diop: Translation at the Service of History». En Agents of Translation, ed. por Bandia, Paul F. y Milton, John. Amsterdam/Philadelphia: John Benjamins.

BANDIA, Paul F. 2014. «Response». The Translator 20 (1): 112-118.

Bárbara CERRATO RODRIGUEZ La (traducción de la) historia como narrativa 
BARILLI, Renato. 1974. Tra presenza e assenza. Milano: Bompiani.

BARNES, Julian. 1989. A History of the World in 10 1/2 Chapters. Londres: Picador.

BARTHES, Roland. [1957] 1999. Mitologías. Madrid: Siglo Veintiuno Editores.

BARTHES, Roland. 1975. The Pleasure of the Text. Traducción de R. Miller. Nueva York: Hill \& Wang.

BARTHES, Roland. 1977. Image, Music, Text. Traducción de S. Heath. Nueva York: Hill \& Wang.

BASSNETT, Susan y André LEFEVERE. 1990. (eds.) Translation, History and Culture. Londres: Pinter Publishers.

BASSNETT, Susan, y André LEFEVERE. 1998. Constructing Cultures: Essays on Literary Translation. Clevedon: Multilingual Matters.

BASTIN, Geoges L. y BANDIA, Paul F. 2006. Charting the Future of Translation Studies. Ottawa: University of Ottawa Press.

BENJAMIN, Walter. [1955] 2005. Tesis sobre la filosofía de la historia y otros fragmentos. Traducción de B. Echeverría. México: Contrahistorias.

BOURDIEU, Pierre. [1985] 2008. ¿Qué significa hablar? Traducción de E. Martínez. Madrid: Akal.

BOURDIEU, Pierre. 1967. "Campo intelectual y Proyecto creador». En Problemas del estructuralismo, ed. por Pouillon, Jean et al. . México: Siglo XXI, 135-182.

BouRdieU, Pierre. 1991. Language and Symbolic Power. Traducción de G. Raymond y M. Adamson. Cambridge (Massachusetts): Harvard University Press.

BOURDIEU, Pierre. 1992. 'Deux impérialismes de l'universel'. En L'Amérique des Français, ed. por Fauré, C. y Bishop, T. París: Éd. François Bourin.

BRAdBuRY, Malcolm. 1983. The Modern American Novel. Oxford: Oxford University Press.

BURKE, Pierre. 1991. (ed.) New Perspectives on Historical Writing. Londres: Polity Press.

D'HAEN, Theo. 1986. "Postmodernism in American Fiction and Art». En Approaching Postmodernism, ed. por Fokkema, Douwe W. y Bertens, Hans. Amsterdam: Benjamins.

DE Certeau, Michel. [1975] 1988. The Writing of History. Nueva York: Columbia University Press.

DE Certeau, Michel. [1986] 2010. Heterologies. Discourse on the Other. Traducción de B. Massumi. Minneapolis: University of Minnesota Press.

DERRIDA, Jacques. [1972] 1977. Posiciones. Traducción de M. Arranz. Valencia: Pre-textos.

ECHEVERRÍA, Bolívar. 2005. La mirada del ángel: en torno a las Tesis sobre la historia de Walter Benjamin. México: Ediciones ERA.

FERGUSON, Russell et al. 1990. (eds.) Out There: Marginalization and Contemporary Cultures. Nueva York: The New Museum of Contemporary Art; Cambridge, Mass., The MIT Press.

FOUCAULT, Michel. [1969] 1972. The Archaeology Of Knowledge. Londres: Vintage.

FOUCAULT, Michel. [1970] 1987. The Order of Things: An Archeology of the Human Sciences. Londres: Vintage.

FOUCAULT, Michel. [1971] 1992. «Nietzsche, la genealogía, la historia». Traducción de J. Varela y F. Álvarez-Uría. En Microfísica del poder. Madrid: La Piqueta.

FOUCAULT, Michel. 1985. La microfísica del poder. Traducción de J. Varela y F. Álvarez-Uría. Madrid: La Piqueta.

GARDINER, Juliet. 1988. (ed.) What Is History Today? Londres: Palgrave MacMillan.

GASS, William H. 1985. Habitations of the Word: Essays. Nueva York: Simon \& Schuster.

Bárbara CERRATO RODRIGUEZ La (traducción de la) historia como narrativa 
GossmaN, Lionel. 1978. «History and Literature. Reproduction or Signification». En The Writing of History. Literary Form and Historical Understanding, ed. por Canary, Robert H. y Kozicki, Henry. Wisconsin: The University of Wisconsin Press, 3-40.

HERMANS, Theo. 1985. (ed.) The Manipulation of Literature. Studies in Literary Translation. Londres: Croom Helm.

HUTCHEON, Linda. 1988. A Poetics of Postmodernism. History, Theory, Fiction. Londres/Nueva York: Routledge.

HUTCHEON, Linda. 1989. «Historiographic Metafiction Parody and the Intertextuality of History». En Intertextuality and Contemporary American Fiction, ed. por O'Donnell, Patrick y Davis, Robert Con. Baltimore: Johns Hopkins University Press, 3-32.

JENKINS, Keith. [1991] 2003. Re-thinking History. Nueva York: Routledge.

KANTZ, Frederick. 1988. (ed.) History from Below. Studies in Popular Protest and Popular Ideology. Oxford: Blackwell.

LEFEVERE, André. 1992. Translation, Rewriting and the Manipulation of Literary Fame. Londres/ Nueva York: Routledge.

LEFEVERE, André y Susan BASSNetT. [1990] 1995. «Introduction: Proust's Grandmother and The Thousand and One Nights: The 'Cultural Turn' in Translation Studies». En Translation, History and Culture, ed. por Lefevere, André y Bassnett, Susan. Londres/Nueva York: Pinter Publishers.

LEITCH, Vincent B. 1983. Deconstructive Criticism: An Advanced Introduction. Nueva York: Columbia University Press.

MORLEY, Neville. 1999. Writing Ancient History. Londres: Duckworth.

MUNDAY, Jeremy. 2014. "Using Primary Sources to Produce a Microhistory of Translation and Translators: Theoretical and Methodological Concerns». The Translator 20 (1).

MUNSLOW, Alun. 2013. Authoring the Past: Writing and Rethinking History. Londres: Routledge.

RIFFATERRE, Michael. 1984. «Intertextual Representation: On Mimesis as Interpretive Discourse». Critical Inquiry 11 (1): 141-162.

SAID, Edward W. 1983. The World, the Text, and the Critic. Cambridge: Harvard University Press. SAID, Edward W. 1986. After the Last Sky: Palestinian Lives. Nueva York: Pantheon.

SPIVAK, Gayatri Chakravorty. [1993] 1996. "Bonding in Difference. Interview with Alfred Arteaga». En The Spivak Reader, ed. por Landry, Donna y MacLean, Gerald. Londres/Nueva York: Routledge.

SPIVAK, Gayatri Chakravorty. 1994. "Can the Subaltern Speak?». En Colonial Discourse and Post-Colonial Theory: A Reader, ed. por Williams, Patrick y Chrisman, Laura. Nueva York: Columbia University Press.

STONE, Lawrence. 1979. «The Revival of Narrative: Reflections on a New Old History». Past and Present 85: 3-24.

SUÁREZ, Luis. 2011. «Franco Bahamonde, Francisco». En Diccionario Biográfico Español, vol. XX. Madrid: Real Academia de la Historia.

TANNER, Tony. 1971. City of Words: American Fiction 1950-1970. Nueva York: Harper \& Row.

THIHER, Allen. 1984. Words in Reflection: Modern Language Theory and Postmodern Fiction. Chicago: University of Chicago Press.

THOMPSON, Edward Palmer. 1966. «History from Below». The Times Literary Supplement, 7 de abril: 279-280.

Bárbara CERRATO RODRIGUEZ La (traducción de la) historia como narrativa 
TYMOCZKO, Maria. 2003. "Ideology and the Position of the Translator: In what Sense is a Translator 'In-between'?». En Apropos of Ideology. Translation Studies on IdeologyIdeologies in Translation Studies, ed. por Calzada-Pérez, María. Manchester: St. Jerome.

TYMOCZKO, Maria y Edwin GENTZLER. 2002. (eds.) Translation and Power. Amherst y Boston: University of Massachussetts Press.

VIDAL, M. ${ }^{a}$ Carmen África. 2013. "The Historian as Translator: Applying Pierre Bourdieu to the Translation of History». En Remapping Habitus in Translation Studies, ed. por Vorderobermeier, Gisella M. Amsterdam: Rodopi, 201-216.

VIDAL, M. ${ }^{a}$ Carmen África. 2017. «Power». En Routledge Handbook of Translation and Culture, ed. por Carbonell, Ovidi y Harding, Sue-Ann. Londres/Nueva York: Routledge.

VIDAL, M. ${ }^{a}$ Carmen África. (en prensa). La traducción y la(s) historia(s).

VON Ranke, Leopold. [1824] 2012. Geschichten der romanischen und germanischen Völker von 1494 bis 1514. Paderborn: Salzwasser Verlag.

WHITE, Hayden. 1978. «The Historical Text as Literary Artifact». En The Writing of History, ed. por Canary, Robert H. y Kozicki, Henry. Wisconsin: The University of Wisconsin Press.

WHITE, Hayden. 1987. The Content of the Form. Narrative Discourse and Historical Representation. Baltimore/Londres: The Johns Hopkins University Press. 